\title{
MicroRNA-124 facilitates lens epithelial cell apoptosis by inhibiting SPRY2 and MMP-2
}

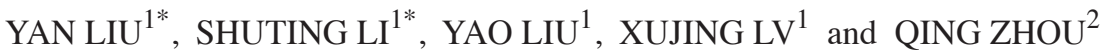 \\ ${ }^{1}$ Department of Ophthalmology, The First People's Hospital of Changzhou, Changzhou, Jiangsu 223000; \\ ${ }^{2}$ Department of Third Institute of Clinical Medicine, Soochow University, Suzhou, Jiangsu 215006, P.R. China
}

Received July 20, 2020; Accepted December 18, 2020

DOI: $10.3892 / \mathrm{mmr} .2021 .12020$

\begin{abstract}
Age-related cataract (ARC) is the primary cause of blindness worldwide. Abnormal expression of microRNAs (miRNAs/miRs) has been reported to be associated with multiple diseases, including ARC. However, the potential role of miR-124 in ARC remains unclear. The present study used the human lens epithelial cell line, SRA01/04, to investigate the potential role of miR-124 in ARC. Reverse transcription-quantitative PCR analysis was performed to detect the expression levels of miR-124, protein sprouty homolog 2 (SPRY2) and matrix metalloproteinase-2 (MMP-2) in ARC tissues, while western blotting was performed to detect the protein levels of SPRY2 and MMP-2. Cell viability and apoptosis of SRA01/04 cells were assessed via Cell Counting Kit- 8 and TUNEL assays, respectively. The interaction between miR-124 and SPRY2 or MMP-2 was confirmed via the dual-luciferase reporter and RNA immunoprecipitation assays. The results of the present study demonstrated that miR-124 expression was significantly upregulated in ARC tissues, and knockdown of miR-124 increased SRA01/04 cell viability and suppressed apoptosis. In addition, SPRY2 and MMP-2 expression was decreased in ARC tissues, and were demonstrated to directly bind to miR-124. Overexpression of SPRY2 or MMP-2 increased SRA01/04 cell viability and repressed apoptosis, the effects of which were reversed following overexpression of miR-124. Taken together, these results suggested that miR-124 facilitates lens epithelial cell apoptosis by modulating SPRY2 or MMP-2 expression, providing a novel treatment approach for ARC.
\end{abstract}

Correspondence to: Dr Yao Liu or Dr Xujing Lv, Department of Ophthalmology, The First People's Hospital of Changzhou, 185 Juqian Street, Tianning, Changzhou, Jiangsu 223000, P.R. China E-mail: liuyao197982@163.com

E-mail: lvxujing78@163.com

*Contributed equally

Key words: microRNA-124, protein sprouty homolog 2, matrix metalloproteinase-2, lens epithelial cells, age-related cataract

\section{Introduction}

Age-related cataract (ARC) is the leading cause of vision impairment and blindness worldwide (1). Surgery is the most effective treatment strategy for cataracts $(2,3)$. However, some postoperative complications may occur, such as posterior capsule opacification $(3,4)$. Previous studies have demonstrated that lens epithelial cell apoptosis is an early event in cataract development $(5,6)$. Thus, determining the underlying molecular mechanism of lens epithelial cells in cataract remains essential.

MicroRNAs (miRNAs/miRs) are a class of small noncoding RNAs $(\sim 22 \mathrm{nt})$ that can modulate the transcription of genes $(7,8)$. Previous studies have reported that abnormal expression of miRNAs is involved in cataracts development $(9,10)$. For example, miR-34 facilitates apoptosis of lens epithelial cells via transforming growth factor (TGF)- $\beta /$ Smads signaling (11). Furthermore, miR-378a modulates reactive oxygen species and the PI3K/AKT pathway in cataract (12), while miR-23b-3p regulates lens epithelial cell apoptosis and autophagy by repressing sirtuin-1 in cataract (13). Recently, miR-124 was reported to repress the viability and invasion of retinoblastoma cells by regulating signal transducer and activator of transcription 3 (STAT3) (14). However, the function of miR-124 in ARC progression remains unclear.

Several studies have suggested that dysregulation of protein sprouty homolog 2 (SPRY2) participates in the progression of different ocular diseases $(15,16)$. For example, Shin et al (17) reported that SPRY2 overexpression in lens cells suppresses TGF- $\beta$-induced epithelial-to-mesenchymal transition (EMT) transition and cataract formation. In addition, Tan et al (18) demonstrated that SPRY2 inhibits the development of lens epithelial cells in anterior subcapsular cataract via the TGF- $\beta$ signaling pathway.

Matrix metalloproteinase-2 (MMP-2) is crucial for retinoblastoma cellular migration and angiogenesis (19). Awasthi et al (20) demonstrated that downregulation of MMP-2 by proteasome suppression decreases the migration of lens epithelial cells and prevents posterior capsular opacification. However, the biological roles of SPRY2 and MMP-2 in $\mathrm{ARC}$ are yet to be investigated.

Thus, the present study aimed to investigate miR-124 expression in ARC tissues and determine whether miR-124 modulates lens epithelial cell apoptosis by regulating SPRY2 and MMP-2. 


\section{Materials and methods}

Clinical samples. A total of 28 ARC anterior capsular tissues (cataracts) and paired normal anterior capsule tissues (without cataracts) were collected from the First People's Hospital of Changzhou (Changzhou, China) between September 2016 and February 2018. The patients included 16 women and 12 men, with a mean age of 63 years (age range, 58-72 years). Based on Lens Opacities Classification System III (21), 28 patients whose lenses with a score of C1-C3, N1-N3, or P1-P3 were enrolled as ARC group, and 28 age-matched individuals who underwent vitrectomy operation of epiretinal membranes were taken as the control group. Patients with complex cataracts with high myopia, ocular trauma, diabetes, and ocular inflammation were excluded from the study. The tissue samples were rapidly frozen in liquid nitrogen and then stored at $-80^{\circ} \mathrm{C}$ prior to subsequent experimentation. The present study was approved by the Ethics Committee of the First People's Hospital of Changzhou and all patients provided written informed consent prior to the study.

Cell culture. The human lens epithelial cell line, SRA01/04, was purchased from the American Type Culture Collection and maintained in Dulbecco's modified Eagle's medium (Gibco; Thermo Fisher Scientific, Inc.) supplemented with 10\% fetal bovine serum (Gibco; Thermo Fisher Scientific, Inc.), $100 \mathrm{U} / \mathrm{ml}$ penicillin (Gibco; Thermo Fisher Scientific, Inc.), and $100 \mathrm{U} / \mathrm{ml}$ streptomycin (Gibco; Thermo Fisher Scientific, Inc.) at $37^{\circ} \mathrm{C}$ in $5 \% \mathrm{CO}_{2}$.

Cell transfection. Short hairpin RNA (shRNA) targeting SPRY2 (shSPRY2; $10 \mathrm{nM}$; 5'-CCUUACCAUUCCUCCACUUTT-3'), shRNA targeting MMP-2 (shMMP-2; $10 \mathrm{nM}$; 5'-GCUGACCUGGAAGAGAACATT-3') and their negative control (shNC; $10 \mathrm{nM}$; 5'-UUCUCCGAAC GUGUC-3'), miR-124 mimics (10 nM; 5'-AACAUUC AACGCUGU CGGUGAGU-3'), NC mimics (10 nM; 5'-UUCUCCGAAC GUGUCACGUTT-3'), miR-124 inhibitor (10 nM; 5'-ACUCACCGACAGCGUUGAAUGUU-3') and NC inhibitor (10 nM; 5'-CAGUACUUUUGUGUAGUACAA-3') were purchased from Shanghai GenePharma Co., Ltd. Cell transfection was performed in SRA01/04 (1 $1 \times 10^{5}$ cells/well) using Lipofectamine $^{\circledR} 2000$ reagent (Invitrogen; Thermo Fisher Scientific, Inc.) for $48 \mathrm{~h}$ at $37^{\circ} \mathrm{C}$ according to the manufacturer's instructions. The full-length of SPRY2 and MMP-2 were subcloned into pcDNA3.1 (10 nM; Shanghai GenePharma Co., Ltd.) to overexpress SPRY2 and MMP-2 levels with empty pcDNA3.1 (10 nM; Shanghai GenePharma Co., Ltd.) serving as the control. The efficiency of transfection was determined in each experiment using reverse transcription-quantitative (RT-q)PCR $48 \mathrm{~h}$ post-transfection. Subsequent experiments were performed at $48 \mathrm{~h}$ post-transfection.

$R T-q P C R$. Total RNA was extracted from ARC tissues and SRA01/04 cells using TRIzol ${ }^{\circledR}$ reagent (Invitrogen; Thermo Fisher Scientific, Inc.), according to the manufacturer's instructions. Total RNA was reverse transcribed into cDNA using the PrimeScript ${ }^{\mathrm{TM}}$ RT Reagent Kit (cat. no. RR047A; Takara Biotechnology Co., Ltd.) according to the manufacturer's instructions. miR-124 expression was detected using the TaqMan ${ }^{\mathrm{TM}}$ MicroRNA kit (cat. no. 4440885;
Applied Biosystems; Thermo Fisher Scientific, Inc.). qPCR was subsequently performed using the TB Green ${ }^{\circledR}$ Premix Ex Taq ${ }^{\mathrm{TM}}$ II detection kit (cat. no. RR820A; Takara Bio, Inc.) and LightCycler ${ }^{\circledR} 480$ Real-Time PCR System (Roche Diagnostics). The following thermocycling conditions were used for qPCR: Initial denaturation at $95^{\circ} \mathrm{C}$ for $3 \mathrm{~min}$; 40 cycles of $95^{\circ} \mathrm{C}$ for $5 \mathrm{sec}$ and $60^{\circ} \mathrm{C}$ for $30 \mathrm{sec}$. Relative expression levels were calculated using the $2^{-\Delta \Delta C q}$ method (22) and normalized to the internal reference genes GAPDH or U6. The sequences of the primers were as follows: miR-124 forward, 5'-GTTGGTGGGAGCTACATTGTCTGC-3' and reverse, 5'- GTGTCGTGGACTCGGCAATTC-3'; SPRY2 forward, 5'-TCGGCAGGTCCCTTTGTCATCC-3' and reverse, 5'-TGCAGGTCAACTGGTGTCGT-3'; MMP-2 forward, 5'-CACTTCCACCACCTCCTGTT-3' and reverse, 5'-TGACCGCTTTCTCCTAGCTC-3'; GAPDH forward, 5'-TGAGCGCGGCTACAGCTT-3' and reverse, 5'-TCCTTAATGTCACGCACGATTT-3'; U6 forward, 5'-ATTGGAACGATACAGAGAAGATT-3' and reverse, 5'-GGAACGCTTCACGAATTTG-3'.

Cell proliferation. The Cell Counting Kit-8 (CCK-8; Dojindo Molecular Technologies, Inc.) assay was performed to assess cell viability. Briefly, SRA01/04 cells were seeded into 96-well plates at $1 \times 10^{4}$ cells per well. Following incubation for $48 \mathrm{~h}$ at $37^{\circ} \mathrm{C}, 10 \mu 1 \mathrm{CCK}-8$ reagent was added to each well and cell viability was subsequently analyzed at a wavelength of $450 \mathrm{~nm}$, using a microplate reader (Thermo Fisher Scientific, Inc.).

Cell apoptosis. The TUNEL assay was performed using the One-Step TUNEL Apoptosis Assay kit (cat. no. C1089; Beyotime Institute of Biotechnology) to assess cell apoptosis according to the manufacturer's protocol. Briefly, SRA01/04 cells $\left(1 \times 10^{4}\right)$ were seeded onto coverslips and fixed with $4 \%$ paraformaldehyde at $4^{\circ} \mathrm{C}$ for $25 \mathrm{~min}$. Cells were subsequently treated with DAPI solution $(300 \mathrm{nM})$ at room temperature for $1 \mathrm{~min}$ and observed in five randomly selected fields of view under a fluorescence microscope (Olympus Corporation; magnification, $\mathrm{x} 200$ ).

Dual-luciferase reporter assay. starbase (starbase.sysu.edu. $\mathrm{cn} /$ ) was used to predict the potential target sequences between miR-124 and SPRY2 and between miR-124 and MMP-2. The mutant sequences of SPRY2 and MMP-2 were generated using a Site-Directed Mutagenesis Kit (cat. no. E0554S; New England Biolabs, Inc.). The wild-type (wt) or mutant (mut) 3'-untranlasted region of SPRY2 or MMP-2 was cloned into a pmirGLO reporter vector (Promega Corporation). The pmirGLO-SPRY2-wt, SPRY2-mut, pmirGLO-MMP-2-wt or MMP-2-mut reporter vector was co-transfected with miR-124 mimics, NC mimics, miR-124 inhibitor or NC inhibitor into SRA01/04 cells ( $1 \times 10^{5}$ cells/well) using Lipofectamine 2000 reagent (Invitrogen; Thermo Fisher Scientific, Inc.). Following incubation for $48 \mathrm{~h}$ at $37^{\circ} \mathrm{C}$, the luciferase activities were detected using a Dual-Luciferase Reporter Assay System (Promega Corporation). Renilla luciferase activity was used as the normalization.

Western blotting. Total protein was extracted from SRA01/04 cells using RIPA buffer. (Thermo Fisher Scientific, Inc.). Total 

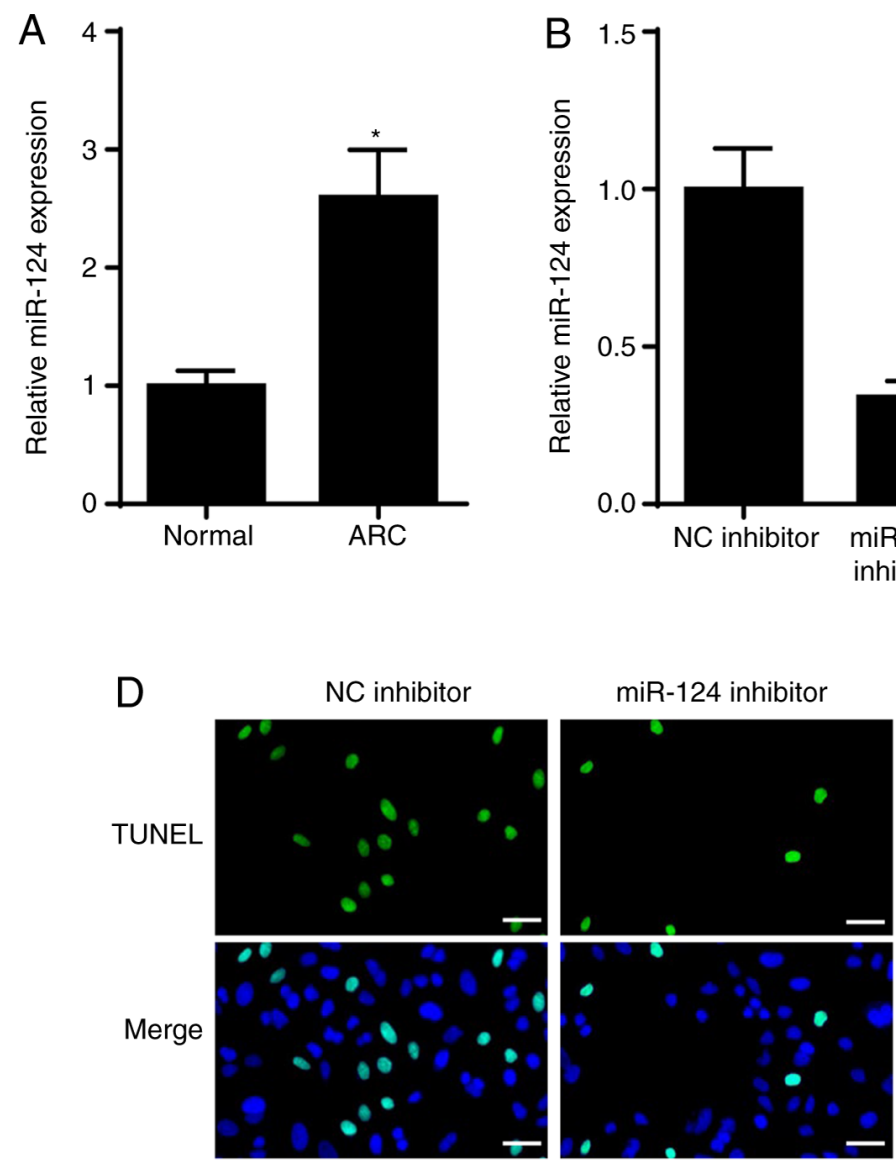
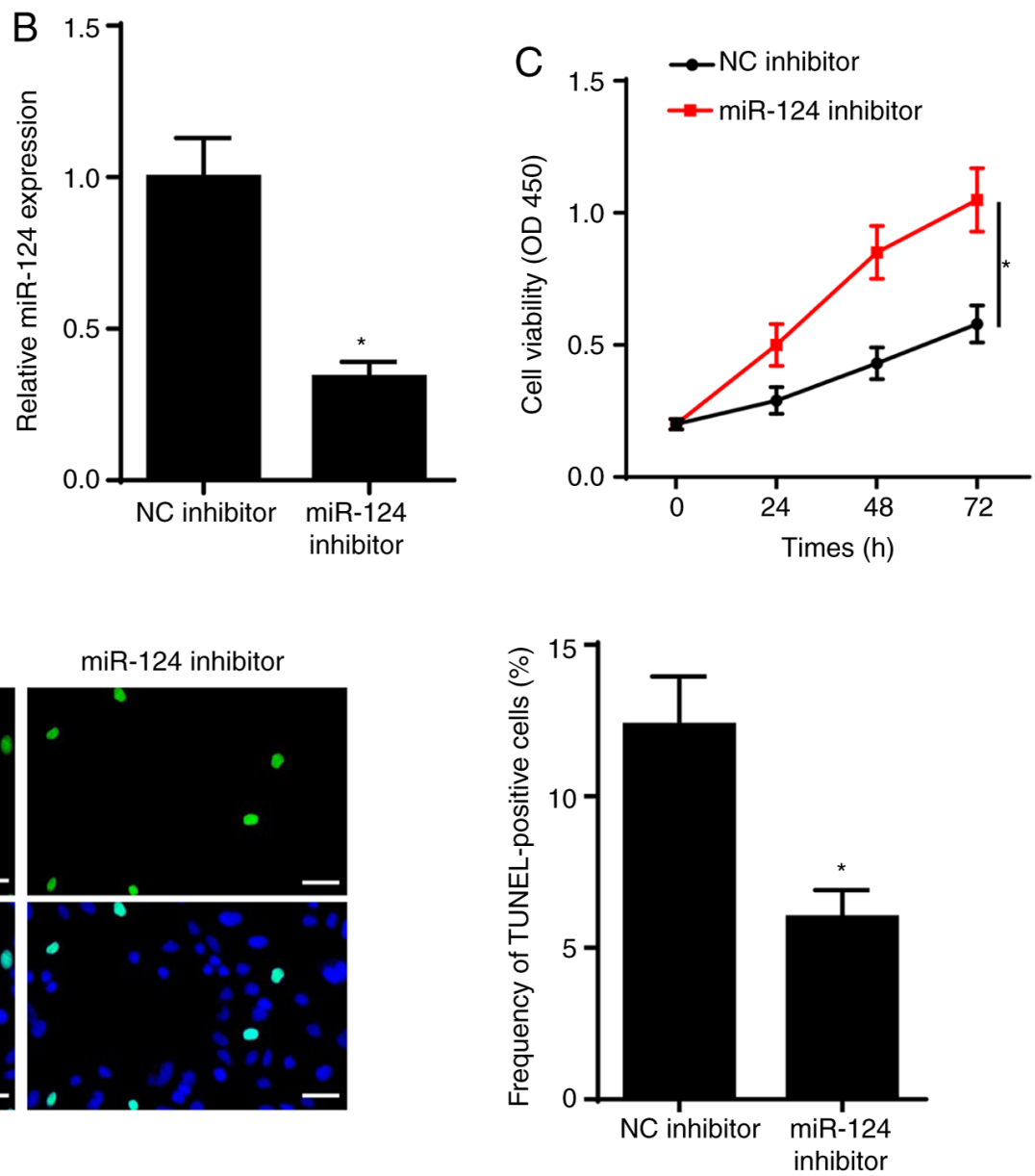

Figure 1. miR-124 expression is increased in ARC tissues and miR-124 inhibitor inhibits SRA01/04 cells apoptosis. (A) RT-qPCR assay was performed to determine miR-124 expression in anterior lens capsules of ARC tissues compared with the normal tissues, $\mathrm{n}=28$. (B) RT-qPCR assay was employed to measure miR-124 expression in SRA01/04 cells transfected with NC inhibitor or miR-124 inhibitor. (C) Cell Counting Kit-8 assay was used to evaluate cell viability in SRA01/04 cells transfected with miR-124 inhibitor or NC inhibitor. (D) TUNEL assay (magnification, x200; scale bar, $50 \mu \mathrm{m}$ ) was performed to analyze cell apoptosis rate of SRA01/04 cells transfected with miR-124 inhibitor or NC inhibitor. * $\mathrm{P}<0.05$ vs. control group. miR, microRNA; ARC, age-related cataract; RT-qPCR, reverse transcription-quantitative PCR; NC, negative control.

protein was quantified using an Enhanced BCA Protein Assay kit (Beyotime Institute of Biotechnology), following which, $50 \mu \mathrm{g}$ protein/lane was separated via SDS-PAGE on $10 \%$ gel (Sigma-Aldrich; Merck KGaA). The separated proteins were transferred onto PVDF membranes (EMD Millipore) and subsequently blocked with $5 \%$ skimmed milk for $1 \mathrm{~h}$ at room temperature. The membranes were then incubated for $12 \mathrm{~h}$ at $4^{\circ} \mathrm{C}$ with the primary antibodies (all Abcam): AntiSPRY2 (1:1,000; cat. no. ab180527), anti-MMP-2 (1:1,000; cat. no. ab92536) and anti-GAPDH (1:1,000, cat. no. ab9485). Following the primary incubation, membranes were incubated with HRP-conjugated secondary antibodies goat anti-rabbit immunoglobin G (IgG; 1:20,000; cat. no. ab205718; Abcam) for $2 \mathrm{~h}$ at $37^{\circ} \mathrm{C}$. Protein bands were visualized using an ECL detection system (EMD Millipore).

RNA immunoprecipitation (RIP). Argonaute 2 (Ago2) RIP was used to determine the interaction between miR-124 and SPRY2 or MMP-2, using the EZ-Magna RIP ${ }^{\text {Tм }}$ RNA-Binding Protein Immunoprecipitation kit (cat. no. 17-701; EMD Millipore). Briefly, SRA01/04 cells $\left(1 \times 10^{5}\right.$ cells/well) were transfected with miR-124 mimics or NC mimics for $48 \mathrm{~h}$ at $37^{\circ} \mathrm{C}$. Cells were subsequently lysed using RIP Lysis Buffer (EMD
Millipore) for $5 \mathrm{~min}$ at $4^{\circ} \mathrm{C}$. After centrifugation $10,000 \mathrm{x} \mathrm{g}$ at $4^{\circ} \mathrm{C}$ for $5 \mathrm{~min}$, cell lysates were conjugated to magnetic beads ( $2 \mu \mathrm{g}$; Thermo Fisher Scientific, Inc.) via rotation, using Ago2 and control IgG (cat. no. ab109761; 1:50; Abcam). The magnetic beads were treated with $0.5 \mathrm{mg} / \mathrm{ml}$ Proteinase $\mathrm{K}$ (EMD Millipore) to digest the protein and then immunoprecipitated RNA was isolated. The immunoprecipitated RNA was analyzed via RT-qPCR analysis.

Statistical analysis. Statistical analysis was performed using SPSS 22.0 (IBM Corp.). All experiments were performed in triplicate and data are presented as the mean \pm standard deviation. Both paired and unpaired Student's t-test were used to compare differences between two groups, while one-way ANOVA, followed by Tukey's post hoc test was used to compare differences between multiple groups. $\mathrm{P}<0.05$ was considered to indicate a statistically significant difference.

\section{Results}

miR-124 expression is upregulated in ARC and miR-124 inhibitor inhibits SRA01/04 cell apoptosis. To investigate the role of miR-124 in ARC, RT-qPCR analysis was performed to 
A Mutant SPRY2:5' UUUUUUAAUAC -GACGGCAUC $3^{\prime}$ Wildtype SPRY2 : $5^{\prime}$ UUUUUUAAUAC -ACAUAUGCA $3^{\prime}$ |||||||| $\mid$ hsa-miR-124 : 3' UACCCUGUAGGAUGUAUACGU 5'
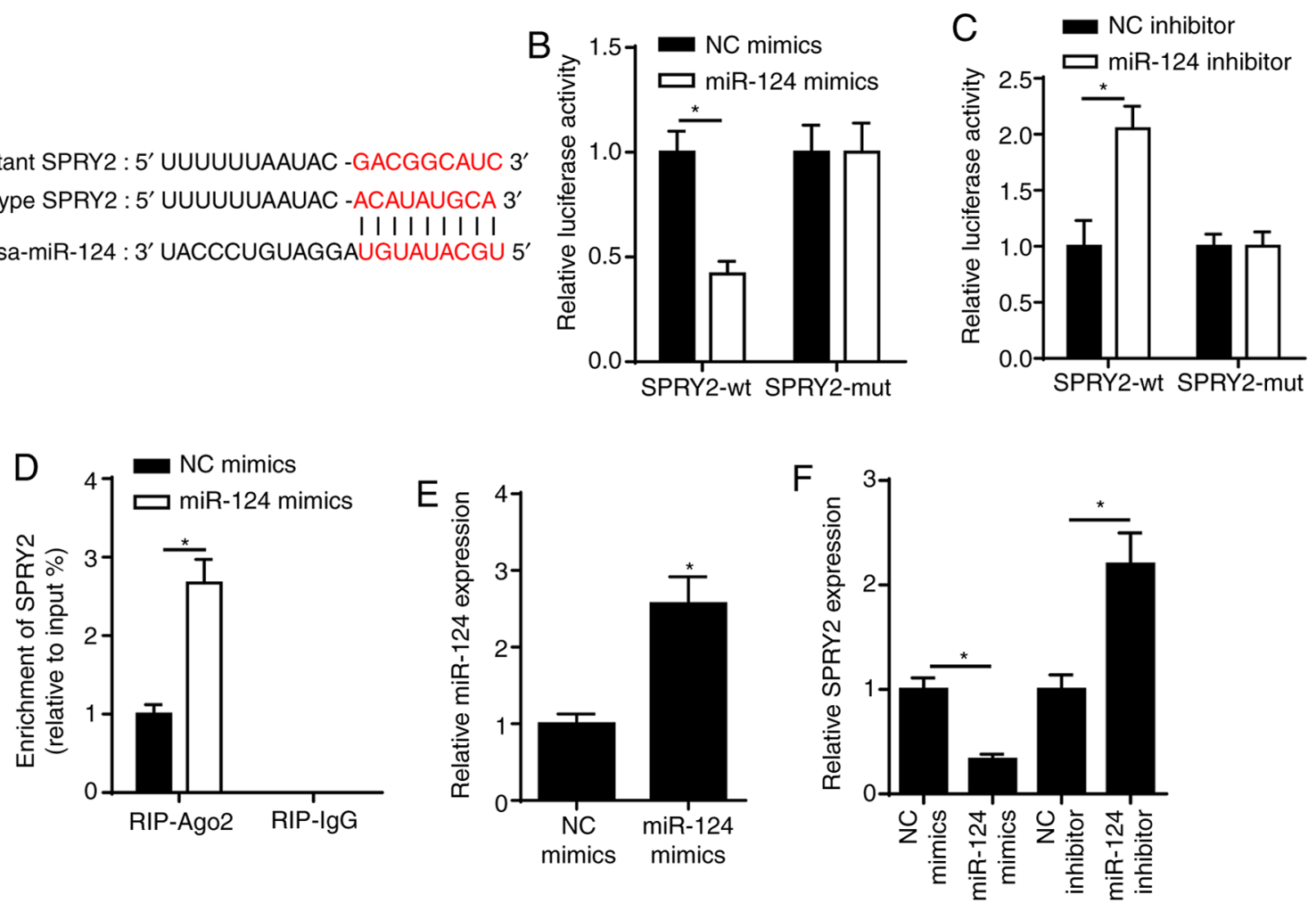

Figure 2. SPRY2 is a target of miR-124. (A) The binding sequence between miR-124 and SPRY2 was predicted using the StarBase website. (B) Dual-luciferase reporter assay showed the luciferase activity of SPRY2-wt or SPRY2-mut in SRA01/04 cells transfected with NC mimics or miR-124 mimics. (C) Dual-luciferase reporter assay showed the luciferase activity of SPRY2-wt or SPRY2-mut in SRA01/04 cells transfected with NC inhibitor or miR-124 inhibitor. (D) RIP assay showed the abundance of SPRY2 enriched by Ago2 or IgG in SRA01/04 cells transfected with NC mimics or miR-124 mimics. (E) RT-qPCR was performed to determine miR-124 expression in SRA01/04 cells transfected with miR-124 mimics or NC mimics. (F) RT-qPCR assay showed the expression of SPRY2 in SRA01/04 cells transfected with NC mimics, miR-124 mimics, NC inhibitor or miR-124 inhibitor. "P<0.05 vs. control group. miR, microRNA; RT-qPCR, reverse transcription-quantitative PCR; NC, negative control; SPRY2, protein sprouty homolog 2; wt, wild-type; mut, mutant; RIP, RNA immunoprecipitation; Ago2, argonaute 2; IgG, immunoglobin G.

detect miR-124 expression in ARC tissues. The results demonstrated that miR-124 expression was significantly increased in the anterior lens capsules of patients with ARC (Fig. 1A). To determine the effect of miR-124 on ARC, SRA01/04 cells were transfected with $\mathrm{NC}$ inhibitor and miR-124 inhibitor. As presented in Fig. 1B, miR-124 expression significantly decreased in SRA01/04 cells transfected with miR-124 inhibitor. The results of the CCK- 8 assay demonstrated that miR-124 knockdown increased SRA01/04 cell viability (Fig. 1C). The results of the TUNEL assay indicated that SRA01/04 cell apoptosis significantly decreased following transfection with miR-124 inhibitor (Fig. 1D). Taken together, these results suggested that miR-124 promoted cell viability and repressed apoptosis of SRA01/04 cells.

SPRY2 is a target of $m i R-124$. To verify the potential molecular mechanism of miR-124 in ARC progression, StarBase was used to predict the putative binding sequences of miR-124 and SPRY2 (Fig. 2A). This interaction was further validated via the dual-luciferase reporter and RIP assays. The dualluciferase reporter assay demonstrated that overexpression of miR-124 significantly decreased the relative luciferase activity of wt-SPRY2; however, no significant changes were observed in the relative luciferase activity of mut-SPRY2 (Fig. 2B). In addition, miR-124 knockdown increased the luciferase activity of wt-SPRY2; however, no significant changes were observed in the luciferase activity of mut-SPRY2 (Fig. 2C). The results of the RIP assay demonstrated that SRA01/04 cells transfected with miR-124 mimics enhanced the enrichment of SPRY2 in the Ago2 group (Fig. 2D). Notably, the addition of miR-124 elevated miR-124 expression in SRA01/04 cells (Fig. 2E). SPRY2 expression was decreased in SRA01/04 cells transfected with miR-124 mimics; however, SPRY2 expression increased in SRA01/04 cells transfected with miR-124 inhibitor (Fig. 2F). Collectively, these results confirmed that SPRY2 was a target of miR-124, and SPRY2 expression was negatively regulated by miR-124.

miR-124 facilitates SRA01/04 cell apoptosis by targeting SPRY2. RT-qPCR demonstrated that the expression of SPRY2 was decreased in ARC (Fig. 3A). To determine whether miR-124 exerts its function by regulating SPRY2, SRA01/04 cells were transfected with shSPRY2, shNC, pcDNA3.1, pcDNA3.1/SPRY2 and pcDNA3.1/SPRY2 + miR-124 mimics. Efficacy analysis demonstrated that SPRY2 mRNA and protein expression levels were significantly decreased in SRA01/04 cells transfected with shSPRY2 (Fig. 3B). Silencing of SPRY2 suppressed SRA01/04 cell viability (Fig. 3C). Moreover, the TUNEL assay determined that knockdown of SPRY2 promoted ARC cell apoptosis (Fig. 3D). Furthermore, SPRY2 mRNA and protein expression levels were increased in SRA01/04 cells transfected with pcDNA3.1/SPRY2 compared 

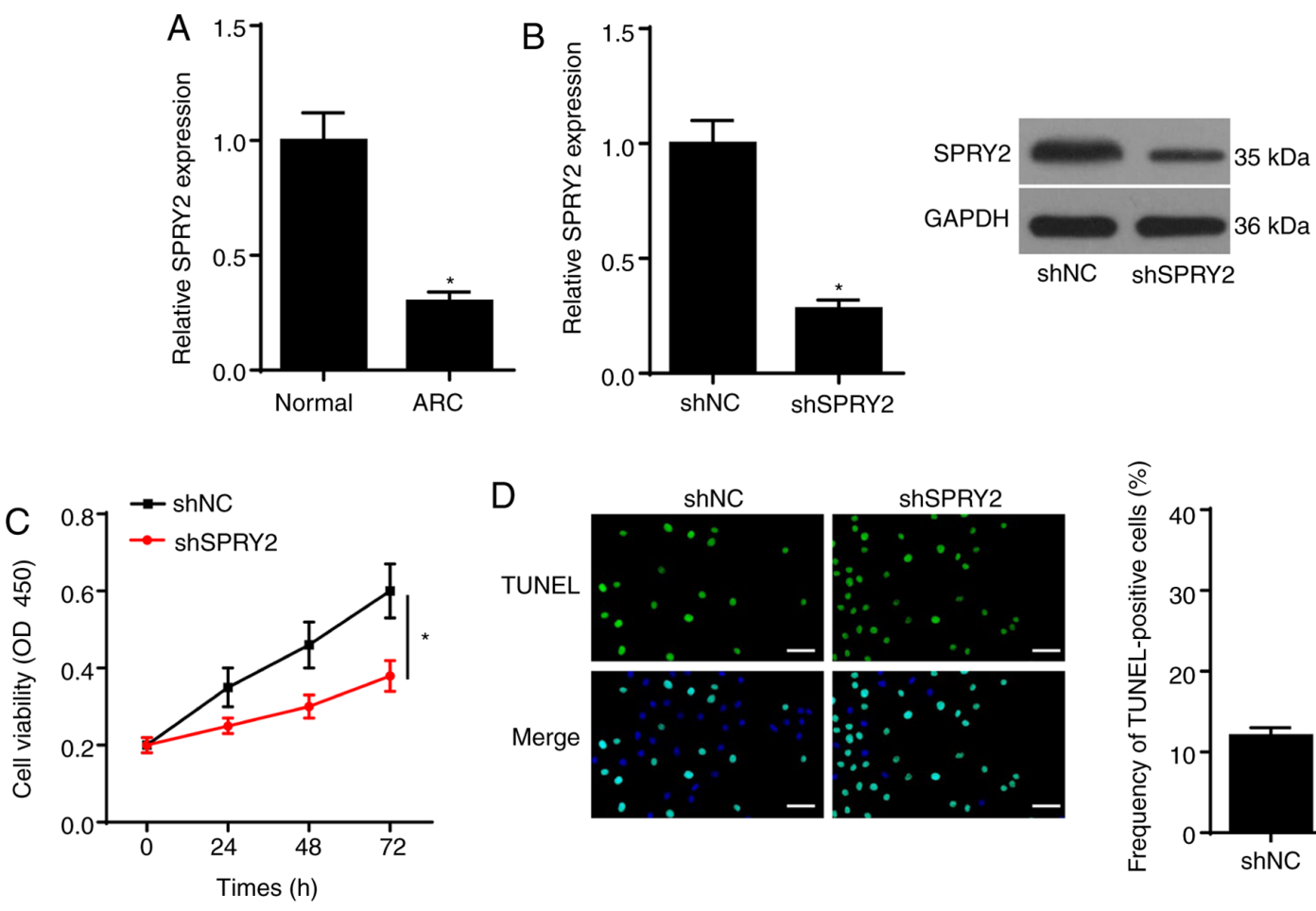

D

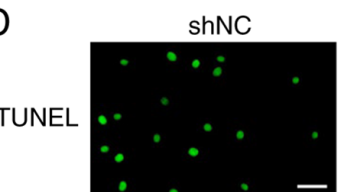

shSPRY2
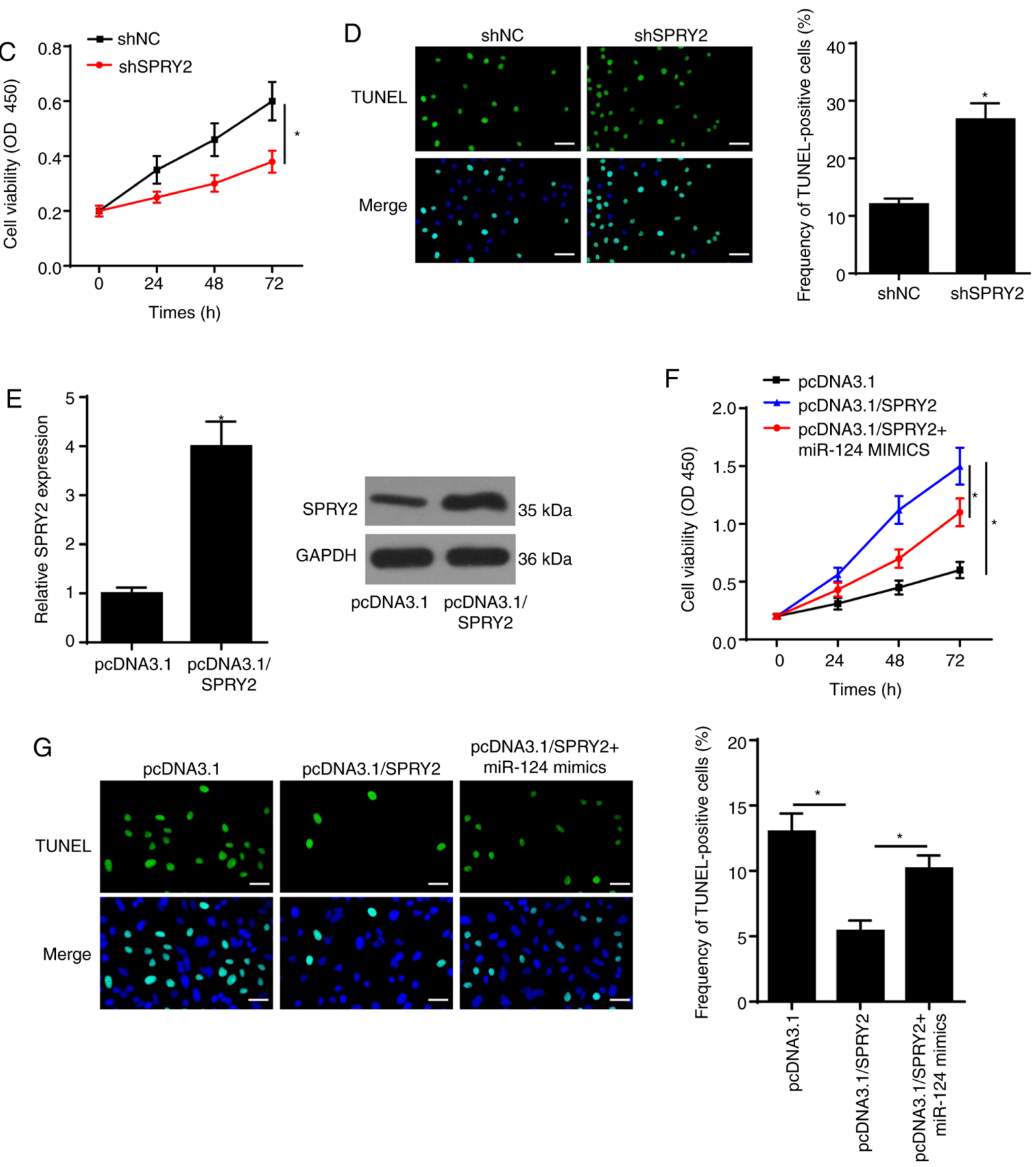

Figure 3. miR-124 increases SRA01/04 cell apoptosis by targeting SPRY2. (A) RT-qPCR assay was employed to assess SPRY2 expression in ARC. (B) RT-qPCR and western blotting assays showed SPRY2 expression in SRA01/04 cells transfected with shSPRY2 or shNC. (C) CCK-8 assay showed the viability of SRA01/04 cells transfected with shSPRY2 or shNC. (D) TUNEL assay (magnification, $\mathrm{x} 200$; scale bar, $50 \mu \mathrm{m}$ ) showed the apoptosis of SRA01/04 cells transfected with shSPRY2 or shNC. (E) RT-qPCR and western blotting assays showed SPRY2 expression in SRA01/04 cells transfected with pcDNA3.1/ SPRY2 or pcDNA3.1. (F) CCK-8 assay showed the proliferation of SRA01/04 cells transfected with pcDNA3.1, pcDNA3.1/SPRY2, pcDNA3.1/SPRY2 + miR-124 mimics. (G) TUNEL assay (magnification, x200; scale bar, $50 \mu \mathrm{m}$ ) showed the apoptosis of SRA01/04 cells transfected with pcDNA3.1, pcDNA3.1/ SPRY2, pcDNA3.1/SPRY2 + miR-124 mimics. "P<0.05 vs. control group. miR, microRNA; RT-qPCR, reverse transcription-quantitative PCR; NC, negative control; SPRY2, protein sprouty homolog 2; sh-, short hairpin RNA; CCK-8, Cell Counting Kit-8; ARC, age-related cataract. 
A Mutant MMP-2: 5' UagcaguUUgCUUUGgCCAUG $3^{\prime}$ Wild-type MMP-2 : 5' UAGCAGUUUGCUUUGUAUGCA 3' hsa-miR-124 : 3' UACCCUGUAGGAUGUAUACGU 5 ,
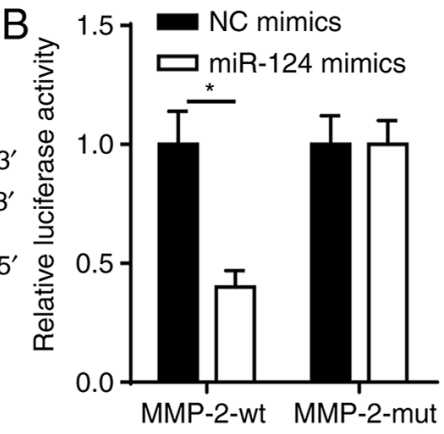

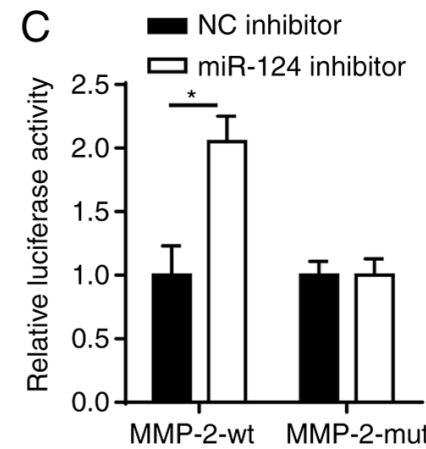

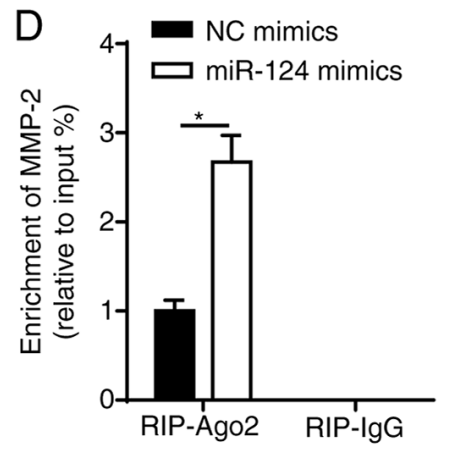

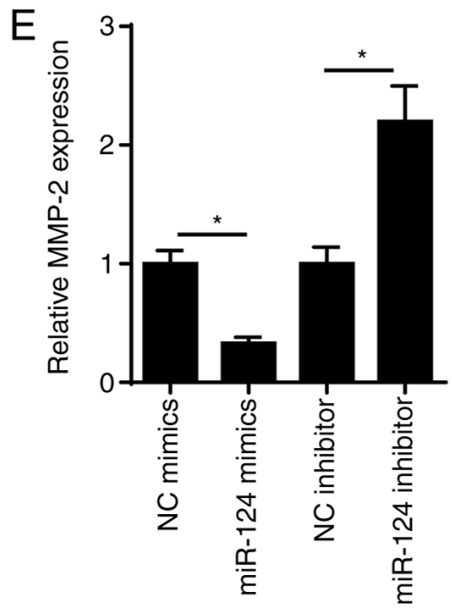

Figure 4. MMP-2 is targeted by miR-124. (A) The binding sequence between MMP-2 and miR-124 was predicted using StarBase software. (B) Dual-luciferase reporter assay showed the luciferase activity of MMP-2-wt or MMP-2-mut in SRA01/04 cells transfected with NC mimics or miR-124 mimics. (C) Dual-luciferase reporter assay showed the luciferase activity of MMP-2-wt or MMP-2-mut in SRA01/04 cells transfected with NC inhibitor or miR-124 inhibitor. (D) RIP assay showed the enrichment of MMP-2 by Ago2 or IgG in SRA01/04 cells transfected with NC mimics or miR-124 mimics. (E) Reverse transcription-quantitative PCR assay revealed MMP-2 expression in SRA01/04 cells transfected with NC mimics or miR-124 mimics, NC inhibitor or miR-124 inhibitor. "P<0.05. miR, microRNA; MMP-2, matrix metalloproteinase-2; wt, wild-type; mut, mutant; RIP, RNA immunoprecipitation; Ago2, argonaute 2; IgG, immunoglobin G; $\mathrm{NC}$, negative control.

with the pcDNA3.1 group (Fig. 3E). In addition, overexpression of SPRY2 increased SRA01/04 cell viability, whereas overexpression of miR-124 reversed its promoting effect on cell viability (Fig. 3F). The results of the TUNEL assay indicated that overexpression of SPRY2 decreased ARC cell apoptosis, the effects of which were reversed following overexpression of miR-124 (Fig. 3G). Taken together, these results suggested that miR-124 regulated SRA01/04 cell functions by targeting SPRY2.

$M M P-2$ is also targeted by miR-124. StarBase was used to predict the binding site between miR-124 and MMP-2 (Fig. 4A). The dual-luciferase reporter assay indicated that the overexpression of miR-124 decreased the luciferase activity of wt-MMP-2 in SRA01/04 cells; however, no significant differences were observed in the luciferase activity of mut-MMP-2 (Fig. 4B and C). These effects were reversed following miR-124 knockdown. In addition, MMP-2 was enriched by Ago2 antibody in SRA01/04 cells transfected with miR-124 mimics (Fig. 4D). RT-qPCR analysis demonstrated that overexpression of miR-124 decreased MMP-2 expression, while transfection with miR-124 inhibitor elevated MMP-2 expression in SRA01/04 cells (Fig. 4E). Collectively, these results suggested that miR-124 targeted MMP-2 in SRA01/04 cells.
miR-124 depends on MMP-2 to regulate SRA01/04 cell apoptosis. To determine the function of MMP-2 in ARC development, RT-qPCR was performed to detect MMP-2 expression in ARC tissues. As presented in Fig. 5A, MMP-2 expression was downregulated in ARC tissues. To further determine whether MMP-2 is a vital effector in miR-124-mediated ARC progression, SRA01/04 cells were transfected with shMMP-2, shNC, pcDNA3.1, pcDNA3.1/MMP-2 and pcDNA3.1/MMP-2 + miR-124 mimics. Transfection of shMMP-2 significantly decreased MMP-2 expression in SRA01/04 cells, at both the mRNA and protein levels (Fig. 5B). Then, the CCK-8 assay showed that MMP-2 knockdown inhibited SRA01/04 cell viability (Fig. 5C). On the contrary, transfection with shMMP-2 promoted ARC cell apoptosis (Fig. 5D). Moreover, overexpression of MMP-2 significantly increased MMP-2 expression in SRA01/04 cells, at both the mRNA and protein levels (Fig. 5E). The results of the CCK-8 assay confirmed that miR-124 can abolish the promotion of MMP-2 overexpression-induced SRA01/04 cell viability (Fig. 5F). In addition, transfection with pcDNA3.1/MMP-2 attenuated ARC cell apoptosis, the effects of which were reversed following overexpression of miR-124 (Fig. 5G). Taken together, these results suggested that MMP-2 was involved in miR-124-mediated SR A01/04 cell apoptosis. 

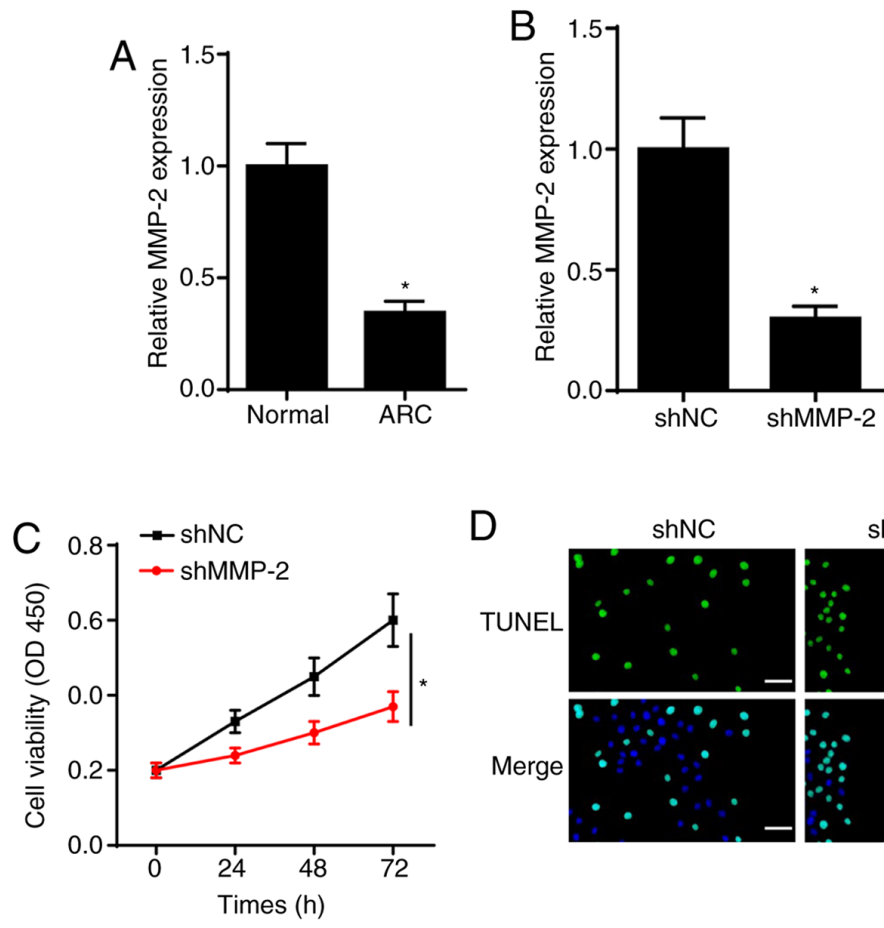

$\mathrm{D}$

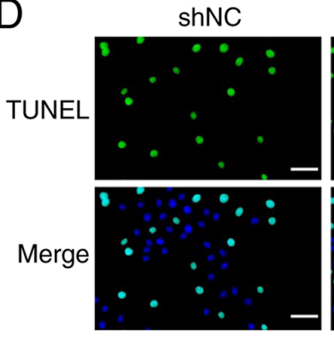

ShMMP-2
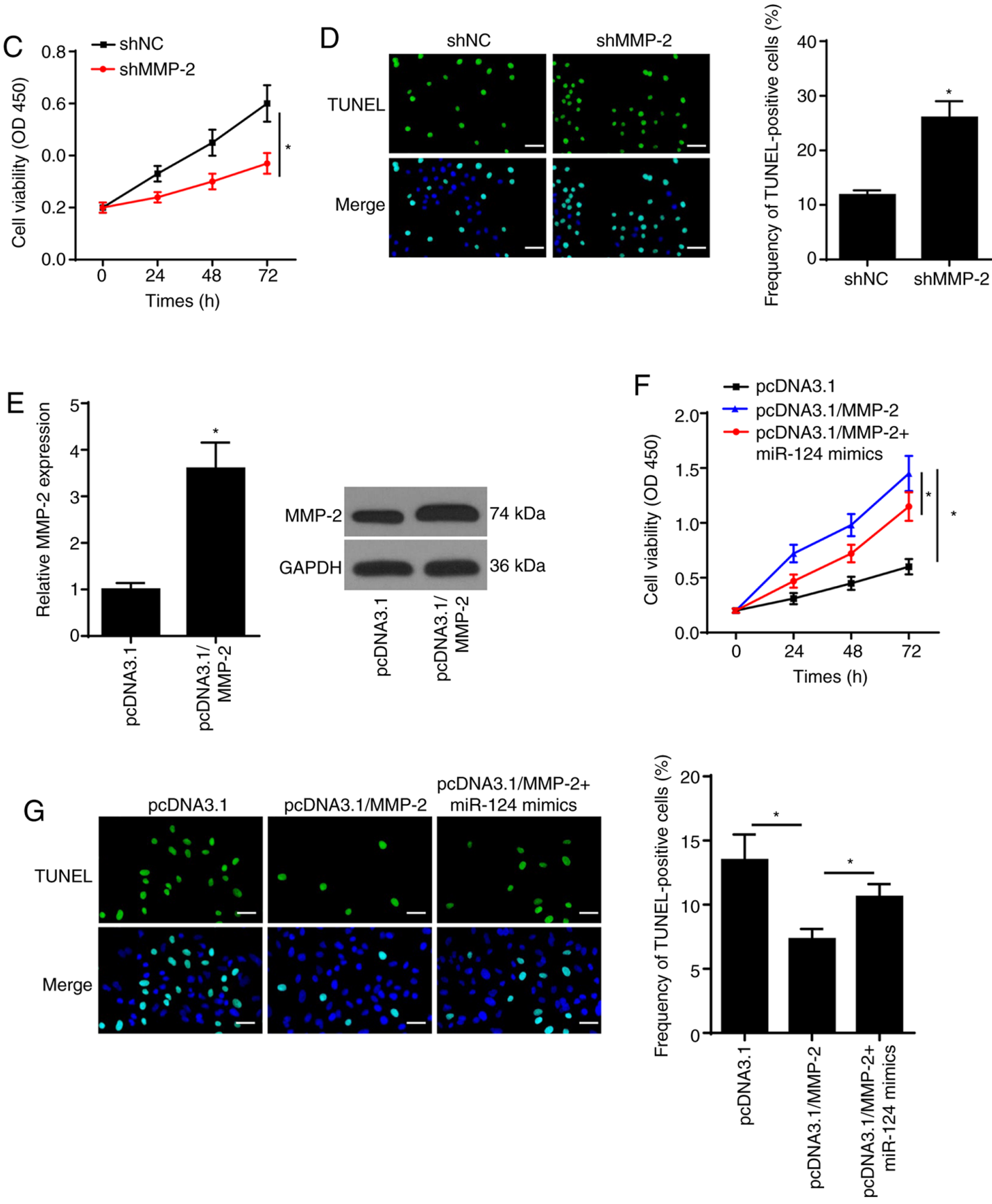

Figure 5. miR-124 depends on MMP-2 to regulate SRA01/04 cell apoptosis. (A) RT-qPCR was used to analyze MMP-2 expression in ARC tissues. (B) RT-qPCR and western blotting assays were performed to determine MMP-2 expression in SRA01/04 cells transfected with shMMP-2 or shNC. (C) CCK-8 assay showed the proliferation of SRA01/04 cells transfected with shMMP-2 or shNC. (D) TUNEL assay (magnification, x200; scale bar, $50 \mu \mathrm{m}$ ) showed the cell apoptosis of SRA01/04 cells transfected with shMMP-2 or shNC. (E) RT-qPCR and western blotting assays were conducted to measure MMP-2 expression in SRA01/04 cells transfected with pcDNA3.1/MMP-2 or pcDNA3.1. (F) CCK-8 assay showed the proliferation of SRA01/04 cells transfected with pcDNA3.1, pcDNA3.1/ MMP-2, pcDNA3.1/MMP-2 + miR-124 mimics. (G) TUNEL assay (magnification, x200; scale bar, $50 \mu \mathrm{m}$ ) showed the apoptosis of SRA01/04 cells transfected with pcDNA3.1, pcDNA3.1/MMP-2, pcDNA3.1/MMP-2 + miR-124 mimics. "P<0.05 vs. control group. ARC, age-related cataract; miR, microRNA; RT-qPCR, reverse transcription-quantitative PCR; NC, negative control; MMP-2, matrix metalloproteinase-2; sh-, short hairpin RNA; CCK-8, Cell Counting Kit-8. 


\section{Discussion}

ARC is one of the most common disorders of the lens worldwide, which leads to reversible blindness $(23,24)$. To the best of our knowledge, the present study was the first to investigate the role of miR-124 in lens epithelial cell apoptosis, and the results demonstrated the interactions between miR-124 and SPRY2 or MMP-2 in SRA01/04 cells. Taken together, these results provided a novel insight into the process of ARC formation.

Increasing evidence suggests that miR-124 participates in several cellular processes, such as proliferation, metastasis, autophagy and apoptosis (25-27). For example, nuclear enriched abundant transcript 1 modulates the viability and apoptosis of retinoblastoma cells by targeting miR-124 (28). Furthermore, silencing of lncRNA XIST suppresses cell proliferation and induces cell apoptosis in retinoblastoma via the miR-124/STAT3 axis (29). The results of the present study demonstrated that miR-124 was upregulated in ARC tissues. The effect of miR-124 on ARC cell viability and apoptosis was also assessed, and the results indicated that miR-124 inhibition promoted SRA01/04 cell viability, but suppressed cell apoptosis.

SPRY2 is a member of the Sprouty family, which exerts essential roles in cellular viability, differentiation and apoptosis $(30,31)$. A recent study reported that miR-23b increases the viability, migration and EMT of lens epithelial cells by targeting SPRY2 (32). The results of the present study demonstrated that SPRY2 expression decreased in ARC, and SPRY2 was confirmed to be a downstream target of miR-124. The results of the CCK-8 and TUNEL assays indicated that SPRY2 facilitated SRA01/04 cell viability and inhibited cell apoptosis, the effects of which were reversed following transfection with miR-124 mimics. Taken together, these results suggested that miR-124 induced apoptosis of SRA01/04 cells by targeting SPRY2.

MMP-2, a member of the MMP family, has been reported to participate in cell processes, including cell proliferation and metastasis $(33,34)$. The results of the present study demonstrated that MMP-2 was downregulated in ARC tissues, and miR-124 inhibited MMP-2 expression by direct interaction. In addition, rescue experiments demonstrated that overexpression of MMP-2 promoted SRA01/04 cell viability, which was attenuated following overexpression of miR-124. Conversely, overexpression of miR-124 reversed the inhibitory effect of overexpressed MMP-2 on SRA01/04 cell apoptosis.

In conclusion, the results of the present study demonstrated that miR-124 expression was elevated in the lens capsule of ARC tissues, and miR-124 regulated SRA01/04 cell viability and apoptosis by targeting SPRY2 and MMP-2. Collectively, these results provided a novel potential therapeutic target for ARC. However, the present study primarily focused on in vitro experiments and the number of clinical samples used was limited. Further in vivo experiments should be performed using an increased number of clinical samples.

\section{Acknowledgements}

Not applicable.

\section{Funding}

The present study was supported by the Youth Project of Jiangsu Natural Science Foundation (grant no. BK20190162), the Applied Basic Research Plan of Changzhou (grant no. CJ20190090), the Applied Basic Research Plan of Changzhou (grant no. CJ20180067) and Young Talent Development Plan of Changzhou Health Commission (2020-233) (grant no. CZQM20200027).

\section{Availability of data and materials}

The datasets used and/or analyzed during the present study are available from the corresponding author upon reasonable request.

\section{Authors' contributions}

YaoL and XL conceived and designed the study. YanL, SL and QZ performed the experiments. YaoL and YanL analyzed the data and drafted the manuscript. SL and XL revised the manuscript. All authors read and approved the final manuscript.

\section{Ethics approval and consent to participate}

The present study was approved by the Ethics Committee of the First People's Hospital of Changzhou (Changzhou, China) and all patients provided written informed consent prior to the study.

\section{Patient consent for publication}

Not applicable.

\section{Competing interests}

The authors declare that they have no competing interests.

\section{References}

1. Nangia V, Jonas JB, George R, Lingam V, Ellwein L, Cicinelli MV, Das A, Flaxman SR, Keeffe JE, Kempen JH, et al; Vision Loss Expert Group of the Global Burden of Disease Study: Prevalence and causes of blindness and vision impairment: Magnitude, temporal trends and projections in South and Central Asia. Br J Ophthalmol 103: 871-877, 2019.

2. Jin C, Chen X, Law A, Kang Y, Wang X, Xu W and Yao K: Different-sized incisions for phacoemulsification in age-related cataract. Cochrane Database Syst Rev 9: CD010510, 2017.

3. Tang Y, Wang X, Wang J, Huang W, Gao Y, Luo Y, Yang J and Lu Y: Prevalence of age-related cataract and cataract surgery in a Chinese adult population: The Taizhou Eye Study. Invest Ophthalmol Vis Sci 57: 1193-1200, 2016.

4. Milazzo S, Grenot M and Benzerroug M: Posterior capsule opacification. J Fr Ophtalmol 37: 825-830, 2014 (In French).

5. Mathias RT, White TW and Gong X: Lens gap junctions in growth, differentiation, and homeostasis. Physiol Rev 90: 179-206, 2010.

6. Belusko PB, Nakajima T, Azuma M and Shearer TR: Expression changes in mRNAs and mitochondrial damage in lens epithelial cells with selenite. Biochim Biophys Acta 1623: 135-142, 2003.

7. Sun T, Li MY, Li PF and Cao JM: MicroRNAs in cardiac autophagy: Small molecules and big role. Cells 7: E104, 2018.

8. Wang J, Haubrock M, Cao KM, Hua X, Zhang CY, Wingender E and Li J: Regulatory coordination of clustered microRNAs based on microRNA-transcription factor regulatory network. BMC Syst Biol 5: 199, 2011. 
9. Yu X, Zheng H, Chan MT and Wu WKK: MicroRNAs: New players in cataract. Am J Transl Res 9: 3896-3903, 2017.

10. Li ZN, Ge MX and Yuan ZF: MicroRNA-182-5p protects human lens epithelial cells against oxidative stress-induced apoptosis by inhibiting NOX4 and p38 MAPK signalling. BMC Ophthalmol 20: 233,2020

11. Zhang GB, Liu ZG, Wang J and Fan W: MiR-34 promotes apoptosis of lens epithelial cells in cataract rats via the TGF- $\beta$ / Smads signaling pathway. Eur Rev Med Pharmacol Sci 24: 3485-3491, 2020.

12. Liu Y, Li H and Liu Y: microRNA-378a regulates the reactive oxygen species (ROS)/phosphatidylinositol 3-kinases (PI3K)/ AKT signaling pathway in human lens epithelial cells and cataract. Med Sci Monit 25: 4314-4321, 2019.

13. Zhou W, Xu J, Wang C, Shi D and Yan Q: miR-23b-3p regulates apoptosis and autophagy via suppressing SIRT1 in lens epithelial cells. J Cell Biochem 120: 19635-19646, 2019.

14. Liu S, Hu C, Wang Y, Shi G, Li Y and Wu H: miR-124 inhibits proliferation and invasion of human retinoblastoma cells by targeting STAT3. Oncol Rep 36: 2398-2404, 2016.

15. Kuracha MR, Burgess D, Siefker E, Cooper JT, Licht JD, Robinson ML and Govindarajan V: Spry1 and Spry2 are necessary for lens vesicle separation and corneal differentiation. Invest Ophthalmol Vis Sci 52: 6887-6897, 2011.

16. Zhang Y, Yuan F, Liu L, Chen Z, Ma X, Lin Z and Zou J: The role of the miR-21/SPRY2 axis in modulating proangiogenic factors, epithelial phenotypes, and wound healing in corneal epithelial cells. Invest Ophthalmol Vis Sci 60: 3854-3862, 2019.

17. Shin EH, Basson MA, Robinson ML, McAvoy JW and Lovicu FJ: Sprouty is a negative regulator of transforming growth factor $\beta$-induced epithelial-to-mesenchymal transition and cataract. Mol Med 18: 861-873, 2012.

18. Tan X, Zhu Y, Chen C, Chen X, Qin Y, Qu B, Luo L, Lin H, Wu M, Chen W, et al: Sprouty2 suppresses epithelial-mesenchymal transition of human lens epithelial cells through blockade of Smad2 and ERK1/2 pathways. PLoS One 11: e0159275, 2016.

19. Webb AH, Gao BT, Goldsmith ZK, Irvine AS, Saleh N, Lee RP Lendermon JB, Bheemreddy R, Zhang Q, Brennan RC, et al: Inhibition of MMP-2 and MMP-9 decreases cellular migration, and angiogenesis in in vitro models of retinoblastoma. BMC Cancer 17: 434, 2017

20. Awasthi N, Wang-Su ST and Wagner BJ: Downregulation of MMP-2 and -9 by proteasome inhibition: A possible mechanism to decrease LEC migration and prevent posterior capsular opacification. Invest Ophthalmol Vis Sci 49: 1998-2003, 2008.

21. Chylack LT Jr, Wolfe JK, Singer DM, Leske MC, Bullimore MA Bailey IL, Friend J, McCarthy D and Wu SY; The Longitudinal Study of Cataract Study Group: The Lens Opacities Classification System III. Arch Ophthalmol 111: 831-836, 1993.
22. Livak KJ and Schmittgen TD: Analysis of relative gene expression data using real-time quantitative PCR and the 2(-Delta Delta C(T)) Method. Methods 25: 402-408, 2001.

23. Thrimawithana TR, Rupenthal ID, Räsch SS, Lim JC, Morton JD and Bunt CR: Drug delivery to the lens for the management of cataracts. Adv Drug Deliv Rev 126: 185-194, 2018.

24. Wei M, Xing KY, Fan YC, Libondi T and Lou MF: Loss of thiol repair systems in human cataractous lenses. Invest Ophthalmol Vis Sci 56: 598-605, 2014.

25. Fu W, Wu X, Yang Z and Mi H: The effect of miR-124-3p on cell proliferation and apoptosis in bladder cancer by targeting EDNRB. Arch Med Sci 15: 1154-1162, 2019.

26. Yan G, Li Y, Zhan L, Sun S, Yuan J, Wang T, Yin Y, Dai Z, Zhu Y, Jiang Z, et al: Decreased miR-124-3p promoted breast cancer proliferation and metastasis by targeting MGAT5. Am J Cancer Res 9: 585-596, 2019.

27. Huang J, Yang Y, Fang F and Liu K: MALAT1 modulates the autophagy of retinoblastoma cell through miR-124-mediated stx17 regulation. J Cell Biochem 119: 3853-3863, 2018

28. Wang L, Yang D, Tian R and Zhang H: NEAT1 promotes retinoblastoma progression via modulating miR-124. J Cell Biochem 120: 15585-15593, 2019.

29. Hu C, Liu S, Han M, Wang Y and Xu C: Knockdown of lncRNA XIST inhibits retinoblastoma progression by modulating the miR-124/STAT3 axis. Biomed Pharmacother 107: 547-554, 2018.

30. Taketomi T, Onimura T, Yoshiga D, Muratsu D, Sanui T, Fukuda T, Kusukawa J and Nakamura S: Sprouty2 is involved in the control of osteoblast proliferation and differentiation through the FGF and BMP signaling pathways. Cell Biol Int 42: 1106-1114, 2018

31. Zhao Q, Chen S, Zhu Z, Yu L, Ren Y, Jiang M, Weng J and Li B: miR-21 promotes EGF-induced pancreatic cancer cell proliferation by targeting Spry2. Cell Death Dis 9: 1157, 2018.

32. Liu W, Yang Y, Yan J and Wang L: MicroRNA-23b-3p promotes the proliferation, migration, and epithelial-mesenchymal transition of lens epithelial cells by targeting Sprouty2. Acta Histochem 121: 704-711, 2019.

33. Sun MX, Yu F, Gong ML,Fan GL and Liu CX: Effects of curcumin on the role of MMP-2 in endometrial cancer cell proliferation and invasion. Eur Rev Med Pharmacol Sci 22: 5033-5041, 2018.

34. Wang X, Yang B, She Y and Ye Y: The lncRNA TP73-AS1 promotes ovarian cancer cell proliferation and metastasis via modulation of MMP2 and MMP9. J Cell Biochem 119: 7790-7799, 2018.

This work is licensed under a Creative Commons Attribution-NonCommercial-NoDerivatives 4.0 International (CC BY-NC-ND 4.0) License. 